\title{
Erratum to: The proliferating brown alga Sargassum polycystum in Tuvalu, South Pacific: assessment of the bloom and applications to local agriculture and sustainable energy
}

\author{
Antoine De Ramon N'Yeurt • Viliamu Iese
}

Published online: 25 January 2015

(C) Springer Science+Business Media Dordrecht 2015

Erratum to: J Appl Phycol

$$
\text { DOI 10.1007/s10811-014-0435-y }
$$

Regrettably, the contents of Tables 1 and 2 were inverted in typesetting. The corrected tables are shown below and in the following page.

Table 1 List of algal species from transects at Fongafale lagoon

\begin{tabular}{lll}
\hline Voucher & Class & Taxa \\
\hline TV13-08 & Chlorophyta & $\begin{array}{c}\text { Caulerpa racemosa } \text { (Forsskå) J. Agardh } \\
\text { var. lamourouxii (Turner) Weber-van } \\
\text { Bosse }\end{array}$ \\
TV13-26 & Caulerpa racemosa (Forsskål) J. Agardh \\
var. typica
\end{tabular}

The online version of the original article can be found at http://dx.doi.org/ 10.1007/s10811-014-0435-y.

A. De Ramon N'Yeurt $(\varangle) \cdot$ V. Iese

Pacific Center for Environment and Sustainable Development, The

University of the South Pacific, Private Mail Bag, Suva, Fiji

e-mail: nyeurt_a@usp.ac.fj
Table 2 List of algal species from transects at the Tuvalu Conservation Area (TCA)

\begin{tabular}{lll}
\hline Voucher & Class & Taxa \\
\hline TV13-12 & Cyanophyta & Schizothrix sp. \\
TV13-13 & Chlorophyta & Caulerpa bikinensis W.R. Taylor \\
TV13-14 & Chlorophyta & Caulerpa cupressoides (Vahl) C. Agardh \\
TV13-24 & Chlorophyta & Caulerpa serrulata (Forsskål) J. Agardh \\
TV13-19 & Chlorophyta & Dictyosphaeria versluysii Weber-van Bosse \\
TV13-16 & Chlorophyta & Halimeda taenicola W.R. Taylor \\
TV13-18 & Chlorophyta & Microdictyon okamurae Setchell \\
TV13-20 & Chlorophyta & Neomeris vanbosseae M.A. Howe \\
\hline
\end{tabular}

